\title{
Agent-Based Advert Placement System for Broadcasting Stations
}

\author{
O. R. Vincent \\ Clausthal University of Technology, Germany \\ and University of Agriculture, Abeokuta, Nigeria
}

\author{
vincent.rebecca@gmail.com
}

\author{
O. Folorunso and A. D. Akinde \\ Department of Computer Science, \\ University of Agriculture, Abeokuta, Nigeria
}

folorunsolusegun@yahoo.com; akinde@yahoo.com

\begin{abstract}
Adverts are used to make services and products known to its likely users or consumers in a very easy and dynamic way. These have become one major medium which business, organization or establishment could function effectively in a competitive environment. Manufacturers and organizations use adverts as a means of reaching the ir intending customers, as regards the goods and services they make available. Adverts therefore serve as agents between organizations and customers. In this paper, a mobile agent based model that would help its users to place timely and effective adverts is described. This is done to aid advert placement in television stations and it is implemented using Nigerian Television stations as case study. Agent moves from one host to another to make enquiry and place adverts. This model is designed with the assumption that each of the host grants access to the mobile agents.
\end{abstract}

Keywords: Static Agents, Advert Placement, Broadcasting Station, E-commerce and Mobile Agents.

\section{Introduction}

The early methods of transmitting information over long distances did not evolve very far distance, until the $19^{\text {th }}$ century, experiments were done to transmit messages via a series of electrical clicks on wires. Thus, the telegraph system was born, laying the foundations for the broadcast of the human voice and other noises. Radio is the first 'modern' media form, and had a huge impact

Material published as part of this publication, either on-line or in print, is copy righted by the Informing Science Institute. Permission to make digital or paper copy of part or all of these works for personal or classroom use is granted without fee provided that the copies are not made or distributed for profit or commercial advantage AND that copies 1) bear this notice in full and 2) give the full citation on the first page. It is permissible to abstract these works so long as cred it is given. To copy in all other cases or to republish or to post on a server or to redistribute to lists requires specific permission and payment of a fee. Contact Publisher@InformingScience.org to request redistribution permission. on the history of the $20^{\text {th }}$ century. People have likened the explosion of radio in the 1920 s to what is happening with the internet today-lots of enthusiasts setting up their 'broadcast slot' and sharing their knowledge with similar people. Wireless communication has really come full circle, as more and more people turn to mobile phones and handle computers that can receive internet 'transaction'. As with the recording of 
images, the broadcast of information is important which has created the need for sending and receiving images along wire less technology. Hence, broadcasting became a reality in the 1920s. By 1928, engineers and had emerged to create a crude receiver set and camera. This went on and even appeared at the world's fair show- the first public viewing of television (TV). Thus, organization and companies begin to understand the commercial opportunities TV station could represent. TV broadcasting in most Countries of the world contributes to global businesses of the Countries through advertisement of company's product.

Advertisement is the set of advertising, given a public notice announcement, which is be ing paid for (Sandeep \& Raghavan, 2005). It could be a public notice of proclamation, notification declaration or broadcast through distance communication with the aim to promote sales. Adverts are place in order to turn the attention of the populace to what is being advertised. On the other hand, adverts are used to make services and products known to its likely users or consumers in a very easy way. Adverts come in different forms, and could be placed in different medium, yet serving one purpose -to reach a group of people in a locality, country, continent or even the entire globe (Alok et al., 1997). Adverts have become one major medium without which any business, organization or establishment could function effectively. Indeed, manufacturers and organizations use adverts as means of reaching their intending customers, as regards the goods and services they make available. Adverts therefore serve as agent between organizations and customers (Guttman \& Meas, 1998a). Mobile agents are the touring clan of agents; they have as an additional attribute, the ability to move from one location to another. Agents have come to have a place in commerce, education, information computation, and communication purposes (Peng et al., 2007).

In this paper, we investigate advert placement in broadcasting stations using Nigeria TV stations as case study and design an agent-based advert placement model for booking advert in the stations. The goal is to enhance information retrieval from te levision stations to place advert using agents and enhancing some necessary communications between these bodies by using mobile agent technology, in order to reduce the network load and optimize the network.

\section{Related Works}

The literature on electronic advertisement has been growing, but only few studies have analyzed how it affect the cooperate organizations. One of which was the work done by Dewan et al. (2002) who designed a balancing content and advertising model to balancing the amount of advertising and content on a web page in order to manage web sites for profitability. The tradeoff was modeled as a control problem for a web site manager who is maximizing the net present value of cash flows by controlling the amount of advertising and content displayed on the web site over its life. The model is calibrated and justified using web site advertisement and audience data. A new measure of web site traffic was developed and was statistically significant in explaining the market value of firms that owned advertising supported web sites.

Another interesting work was the one of Chickering and Heckerman (2003) who presented a targeted advertising model on the web using inventory management with the aim that and company who maintains websites can make considerable revenue by running advertisements, which therefore compete to attract advertisers. If advertisers can be attracted when companies deliver high click-through rates on a site which increases revenue directly, consequently, companies can benefit from de livery systems that display advertisements selectively to those visitors most like ly to click though. A delivery system that maximizes click-through rate given inventory-management constraints in the form of advertisement quotas was developed. The system uses predictive segments in conjunction with a linear program to perform the constrained optimization. Using a real web site, they demonstrated the efficacy of the system by finding revenue-optimal advertisement schedules under a wide variety of pricing models. 
Fruend and and Noar (2004) identified advertisement placement problem as the space and time sharing problem on the Internet. The research considered a web page containing a rectangular display area in which advertisements may appear. The display area was utilized by allowing several small ads to appear simultaneously side by side, as well as by cycling through a schedule of ads, allow ing different ads to be displayed at different times. A customer wishing to purchase advertising space specifies an ad size and a display count, which is the number of times their ad should appear during each cycle. The scheduler may accept or reject any given advertisement, but must be able to schedule all accepted ads within the given time and space constraints. With an objective to schedule a maximum-prof it subset of ads, an approximation algorithm for the general problem, as well as approximation algorithms for the two special cases described.

Sandeep and Raghavan (2005) also considered advertising as a critical process for promoting both products and services in global trade. They address problems faced by online advertisement service providers by proposing a multi-s lot and multi-site combinatorial auction for allocating scarce advertisement slots available on multiple sites. They observe that combinatorial auctions serve as effective mechanisms for allocating advertising slots over the internet. They solved the NP-hard combinatorial optimization problem which involves winner-determination in multi-item and multi-unit combinatorial auctions through allocation of advertising space by a web service provider.

Zhou et al. (2008) presented a mechanism for on-line advertisement placement to Deter click fraud. Investigations were made on the principal-agent problem of the on-line advertising market with respect to the publisher, the coordinator, and the advertiser. They categorised the publishercoordinator contract as a double-sided moral hazard problem and the coordinator-advertiser contract as a double moral hazard problem with the agent's effort observed. Their findings were compared to that of the auction-based model. The cause and conditions of click fraud under both models were dealt with given detailed explanations. Using the ir model, they were able to achieve an efficient market deters click fraud.

Goldfarb and Tucker (2008) worked on research advertisement based on economic and business dimension. They examined a profitable side of the long tall of advertising that is not possible under the traditional broadcast advertising model. They provided clear empirical evidence of the extent to which advertis ing context based advertisement has ability to target very narrow markets which provides 'ling tail of advertising' that is not feasible under the traditional broadcast advertising model. It was found out that search engine advertising is most valuable when firms have just a few hard-to-reach customers.

\section{Agent-Based Advert Placement}

Advertisement is believed to be a major factor behind any successful business. The world has turned into a global village in which every individual and or organization depends on an electronic wizard to assist in carrying out their numerous tasks. This results from the reliability, accuracy, speed, effectiveness and ability to process large information among other strength, which the computer system alongside the Internet facilities provides. Different forms of electronic means are used these days to enhance businesses via the Internet (Dasgupta et al., 1998). This varies from different forms of e-commerce, which include trading on-line, online advertisements, electronic payment system, to name but a few. Mobile agent technologies can be used to automate several of the most time consuming stages of the buying, negotiating, and online decision processes. Unlike "traditional" software, mobile agents are personalized and autonomous. They move around the network, searching for a user specified product or services across different places in different locations. With the mobility of agents, information exchange is local and does not cover the network, thus saving network latencies and load (Dasgupta et al., 1999). 
We consider a case where the user has a Personal Digital Assistant (PDA) which he periodically connects to the network for a relatively short time in Figure 1. He want to place advert for company in a television station and sends agent out to negotiate from different stations and place advert in an appropriate station. The user want this advert to be places in a time called marketing time. Some of the important attribute the user want for the advert include the timing of the advert, the price of the advert, the time duration, etc. The agent will have to exchange information and negotiate to be able to place this advert according to the overall requirements of the user. After the agents are sent into the network, the user disconnects his PDA from the network. There is a semi-trusted platform which keeps track of the different mobile agents. The platform is given information needed during the negotiation.

The agents travel and collect information from different television station host and negotiate for pricing, the agent then take its decision on which station to place the advert. The agent communicate from one host to another service for the best station it could place its advert. Communication between hosts on the Internet consumes network latencies and increases the network load (Danny \& Mitsuru, 1999). However, transfer of information between different host can be optimized through the use of mobile agents. These agents can function with more flexibilities, reduced network bandwidth and latencies (Aderounmu, 2004).

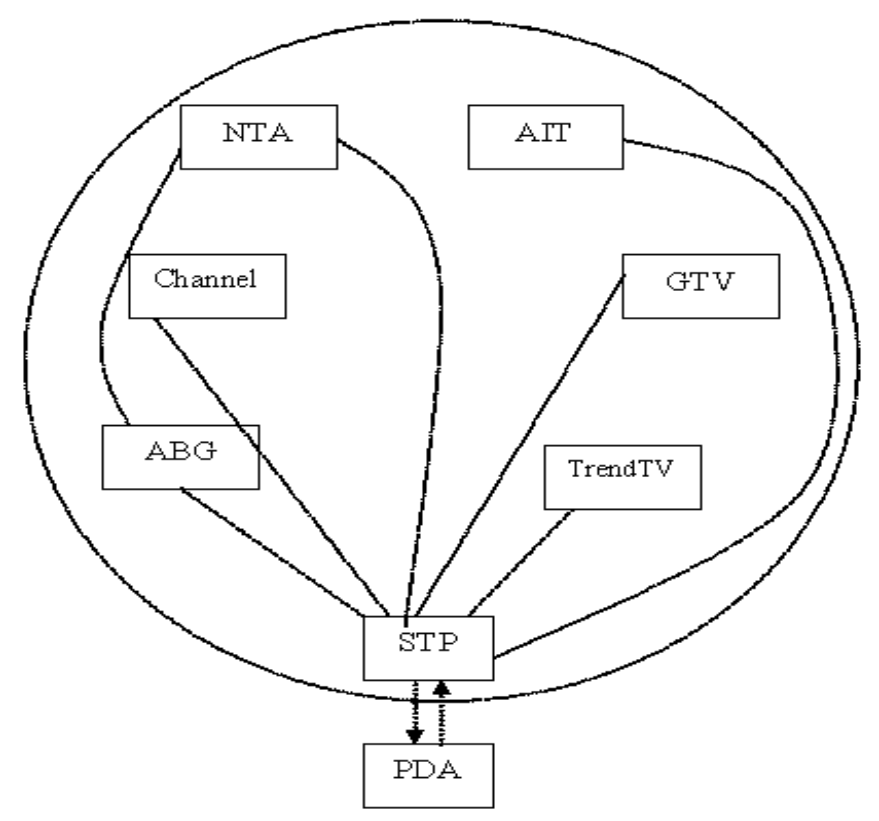

Figure 1: An Agent-based Scenario for Advert Placement in Broadcasting Stations

There are different types of adverts: jingles, sponsorship, backdrops, and scroll messages. Adverts booking are mostly done traditionally though, some stations permit online bookings. Interested bodies could visit stations to make their advert bookings. Depending on agreement reached, such adverts are booked or considered for booking. For the purpose of this work, ten television stations in Nigeria are considered case studies. The TV station are: Nigeria Television Station (NTA), ABG Communications Limited, Africa Independent Television (AIT), Cable Mission Television Network (CMTV), Channels Television, Communication Trends Nigeria Limited (CTL.TV), Cable Station using M.M.D.S Channels, Gateway Television (GTV), Communications Limited and Multi-choice Nigeria Limited (Direct to Home Television - Dth). 


\section{The Model}

The agent-based advert placement system application is modeled to enable a user send agents to book advert in TV station on behalf of the user. The agent visits different televis ion stations via network routes, make enquiries, take a deciding by booking the advert in a desired station and reports back to its home host. The work of the knowledge based agent is to make enquiry about advert placement in the stations, compare the response gotten from these station and choose the best station to book advert. The agent simulation model for advert placement is shown in Figure 2 .

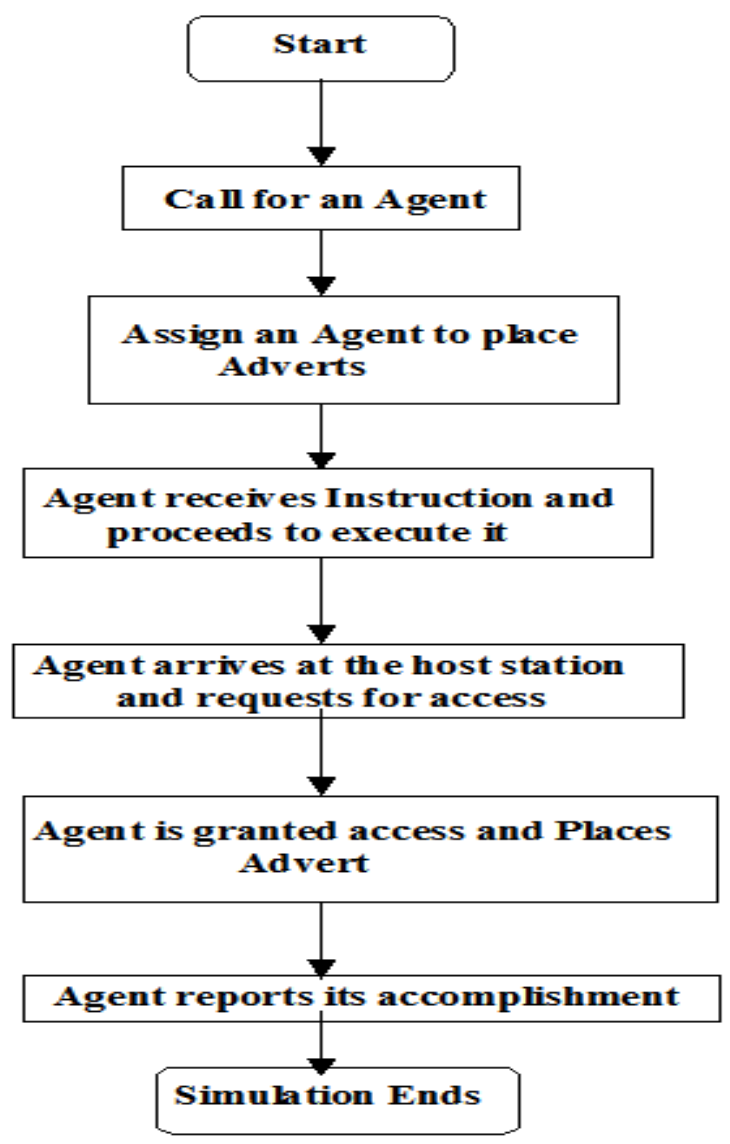

Figure 2: Agent-Based Advert Placement Simulation Process

We investigated advert efficiency as a load instrument; a simulation of an agent accepting foreign agents to check for an appropriate advert placement in a broadcasting station. Figure 3 shows the simulation execution of each server.

\section{A Single Server Point of View}

Assumed a single site agents, the simulation was implemented in AgentSheet with JAVA 1.5.1 in Pentium M 512 of RAM PC with numerical routines. The simulation of visiting agents in home server is shown in Figure 3. 


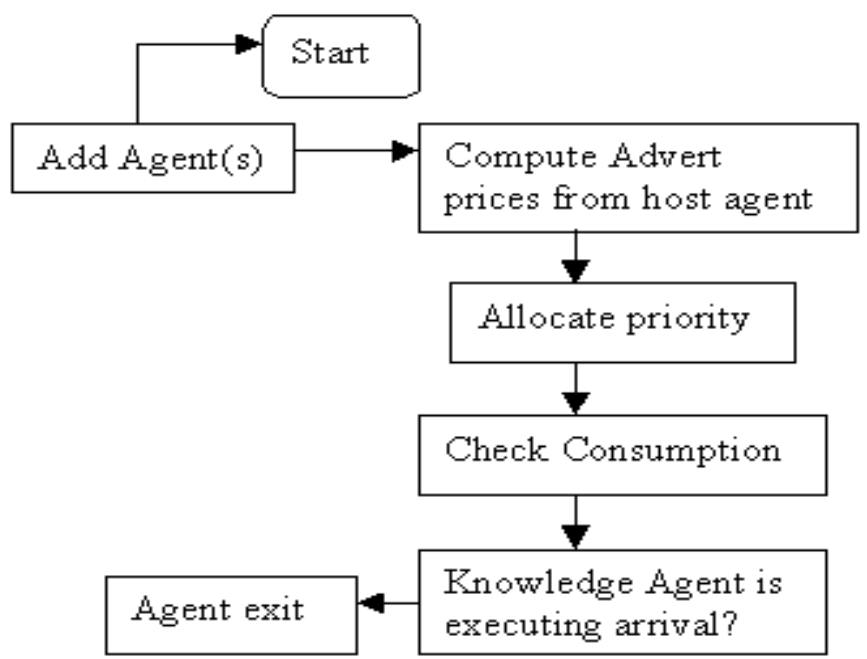

Figure 3: Simulation of Visiting Agents in Home-based Server.

In our simulation, the server allocates execution time to incoming agents by searching the aggregate demand for a market price; clearing every time an agent enters or exits the system. Since Poisson arrival rate with exponential job sizes Q are distribution standard in queuing processor time, we therefore assumed that agents arrived at this rate. Each agent has an exponentially distributed number of destination hops in its itinerary. Agents were designed to represent these stations with features depictions similar to that of their respective logos. The design represents agent SpecialDuties, that receive instructions directly from the user and then passes it on to AgentBookAdvert, which migrates from one host to another to place adverts. The whole process of the design begins with the creation of a project, creation of agents, editing of agent depictions and giving agents behavioral rules. The algor ithms for our agent-based advert placement are as follows.

\section{Algorithm for agent-based advert placement}

Input: Searching Agent

Output: Agent Placed advert

Step 1: A new project created and named Agent-Based Advert Placement.

Step 2: Declaration of agent size, e.g. customer size of $24 \times 24$ chosen

Step 3: Opened gallery to select agent, Edit its depiction, Create other agents and edit the ir depictions.

Step 4: Edit Agents behaviour. The default behavior of each agent is "if no condition then no action". Assign appropriate behaviors to each agent so as to function according to the design model

Step 5: Design the simulation environment

Step6: Run simulation; implementing step 1 to 3 gives Figure 4. 


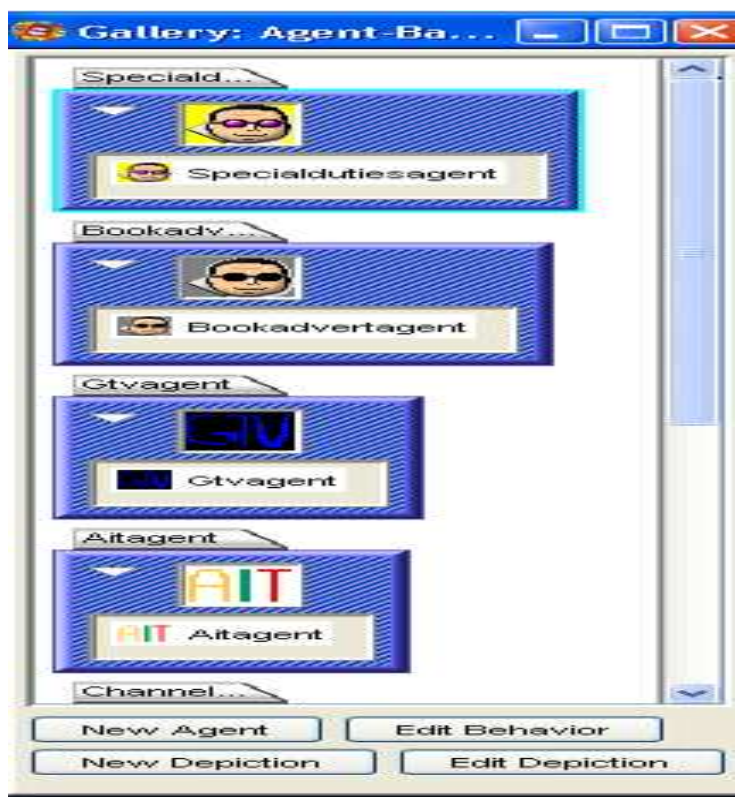

Figure 4: Agents Creation for Television Stations

\section{Design Features}

The simulated agents and environment designed for this implementation exhibits the following features:

1. Two categories of agents; the stationary agents and the mobile agents. Here the stationary agents are the television stations agents, which were not coded to exhibit movement, while the mobile agents are the SpecialDutiesAgent and the BookAdvertAgent.

2. Interaction between agents is enhanced.

3. Unique identities: the agents all possess different behavioral characteristics. The simulated agents react differently to different actions.

4. Depicts an environment in which the agent host allows agents to communicate with one another.

5. An environment where agents interact but do not interfere with one another.

6. Depicts an agent that moves from one network to another in a network environment, yet with complete control of its movement.

As an illustration of the implementation, we consider an AgentBookAdvert that is required to visit NTA, GTV, AIT site, etc. As soon as the AgentBookAdvert arrives near the station GTV's host in Figure 5, permission to ga in access is also requested for and it was granted. The Agent BookAdvert thereafter carries out its mission and later thank the station's agent. 


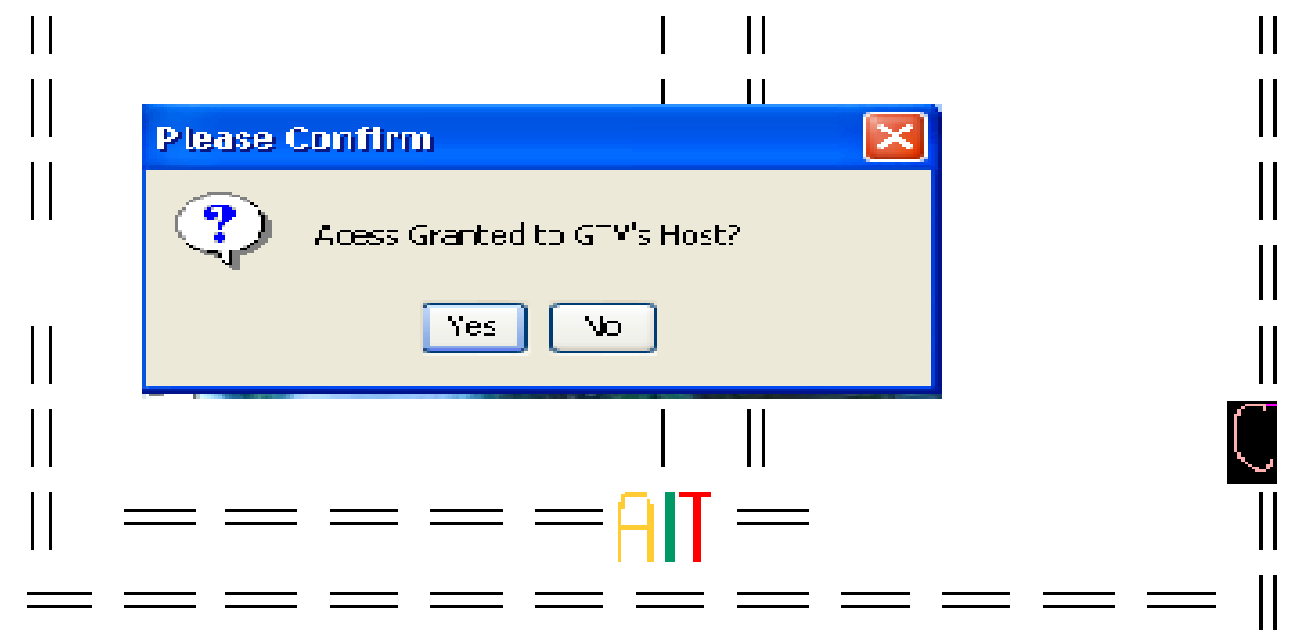

Figure 5: Gallery showing AgentBookAdvert Arriving at GTV's Host

As soon as the AgentBookAdvert accomplished its aim with the station GTV, it moves to the station Channels, requests for access, gains access and concludes its mission.

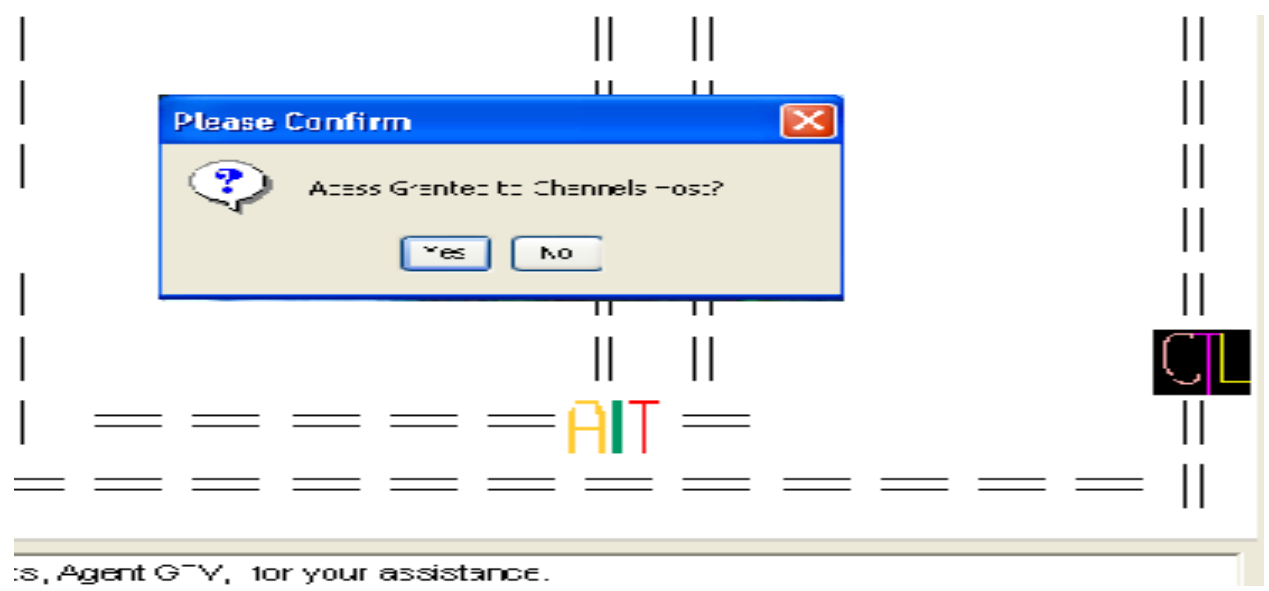

Figure 6: Galle ry showing Agent BookAdvert Arriving at Channel'sHost

The AgentBookAdvert leaves the station Channels host, arrives the station CTL's host, requests for access in figure 6, gains access to make its enquiry. After getting concrete information about the booking and at what rate, the agent decides which TV station it should book advert, at what and what time the advert should be placed. After placing adverts in the televis ion station, the AgentBookAdvert returns to its initial location and reports to its fellow agent, AgentSpecialDuties.

\section{Conclusion}

A study on advert bookings and placements in broadcasting stations, a case study of Nigeria Television station is presented. Advertisement is very important to organization and cooperate body but the problem has been where, what time and at what rate advert should be booked. Most organizations want there adverts to be placed at a time called "hot time", when it is expected the message will be passed across millions of people.

In this paper, we have proposed an agent-based advert booking and placement model with the aim that mobile agents could enhance advert placement in TV stations. It is implemented to aid advert placements with the view of optimizing network bandwidth and latency. In the Implementation, 
mobile agent moves from one TV host to another to make enquiries and adverts booking. The model is designed with the assumption that each TV host grants access to the mobile agents. The agent has the capabilities to function effectively in a market environment with various activities ranging from information retrieval, tickets booking, purchasing and advert placement.

\section{References}

Aderounmu, G. A. (2004). Performance comparis on of re mote procedure calling and mobile agent approach to control and data transfer in distributed computing environment. Journal of Network and Computer Applications, 27, 113-129.

Adler, M., Gibbons, P., \& Matias, Y., (2002).Scheduling space-sharing for internet advertising. J. Scheduling,5(2), 103-119.

Alok, G., Dale O. S., \& Andrew, B. W. (1997). Priority pricing of integrated services networks. In L. W. McKnight \& J. P. Bailey (Eds.), Internet economics (pp. 323-352). Ca mbridge, MA: MIT Press.

Chavez, A., \& Maes, P. K. (1996). An agent marketplace for buying and selling goods. Proceedings of the First International Conference on the Practical Application of Intelligent Agents and Multi-Agent Technology, London, UK, 75-90.

Chickering, D. M., \& Heckerman, D. (2003). Targeted advertising on the web with inventory management. Interfaces, 33(5), 71-77.

Danny, B. L., \& Mitsuru, O. (1999). Seven good reasons for mobile agents. Communications of the ACM, $42,3$.

Dasgupta, P. N., Narasimhan, L. E., \& Melliar-Smith, P. M. (1998). A supplier driven electronic marketplace using mobile agents. Proceedings of the First International Conference on Telecommunications and E-Commerce, Nashville, TN.

Dasgupta, P. N., Narasimhan, L. E., \& Melliar-Smith, P. M. (1999). MAgNET: Mobile agents for networked electronic trad ing. IEEE Transactions on Knowledge and Data Engineering, Special Issue on Web Applications.

Dave, S., \& Bart, P. (2006). Secure e-commerce using mobile agents on untrusted hosts. COSIC Internal Report.

Dewan, R., Freimer, M., \& Zhang, J. (.2002). Managing web sites for profitability: Balancing content and advertising. Proceedings of the 35th Annual Hawaii Intemational Conference on System Sciences (HICSS'02)-Volume 7, p.181, January 07-10.

Doorenbos, R. O., \& Weld, D. (1997). A scalable comparison/shopping agent for the world wide web. Proceedings of the first International Conference on Autonomous Agents, Marina del Rey, CA.

Freund, A., \& Naor, J. (2004). Approximating the advertisement placement problem. Journal of Scheduling, 7(5), 365-374.

Goldfarb, A., \& Tucker, C. (2008). Econo mic and business dimensions search engine advertising. Communications of the ACM, 51(11), 22-24.

Guttman, R. A., \& Maes, P. (1998a). Agent-med iated electronic commerce: A survey. The Knowledge Engineering Review, 13(2), 147-159.

Guttman, R. A., \& Maes, P. (1998b). Agent-med iated integrative negotiation for retail electronic commerce. Mediated Electronic Trading Workshop, Minneapolis, MN.

Maes, P., Robert, H. G., \& Ale xandros, G. M. (1999). Agents that buy and sell. Communications of the ACM, 42(3), 81-91.

Muller, J. P. (1996). A cooperation model for autonomous agents. The third Workshop on Agent Theories, Architectures, and Languages (ATAL' 96), Budapest, Hungary, pp. 245-260. 
Peng, L., Manghuui, T., Yen, I., \& Zhonghang, X. (2007). Preference update for e-commerce applications: Model, language, and processing. Electron Commerce Res. 7(1), 17-44.

Rahul, J., \& Sridhar, I. (2005). Mobile agents for e-commerce. KR School of Information Technology, Indian Institute of Technology, Bombay, Roll No.: 99329011.

Sandeep D., \& Raghavan, N. R. S. (2005). Allocation of advertising space by a web service provider using combinatorial auctions. Sadhana, 30(2 \& 3), 213-230.

Saurab, N., Su mit, C., \& David, K. (1996). An RPC mechanism for transportable agents. Dart mouth PCSTR96-280, Department of Computer Science, Dart mouth College.

Song, R., \& Korba, L. (2003). Security communication architecture for mobile agents and e-commerce. Proceedings of the International Workshop on Mobile Systems, E-Commerce and Agent Technology (MSEAT'2003). Miami, Florida, USA.

Tsvetovatyy, M. B., Mobasher, M. G., \& Wieckowski, Z (1996). MA GMA: An agent-based virtual market for electronic co mmerce. Applied Artificial Intelligence, 11(6), 501-523.

Westhoff, D., Schneider, M., Unger, C., \& Kaderali, F. (1999). Methods for protecting a mobile agent's route. Proceedings of International Information Security Workshop'99 (ISW'99), LNCS 1729, pp. 57 71 .

Zeng, D., \& Sycara, K. (1996). How can an agent learn to negotiate? The Third Workshop on Agent Theories, Architectures, and Languages (ATAL'96), Budapest, Hungary, pp. 233-244.

Zhou, W., Bandyopadhyay, S., Cheng, H., \& Pathak, P. (2008). A mechanism for on-line advertisement placement to deter click fraud. International Journal of Electronic Commerce, 13(2), 9-28

\section{Biographies}

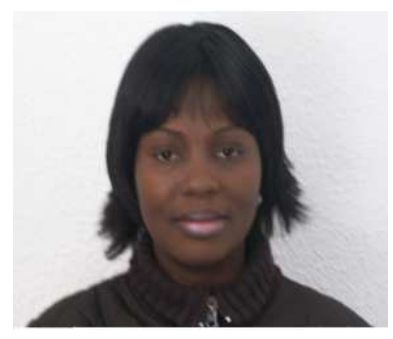

Rebecca Vincent is a Lecturer in the Department of Computer Science, University of Agriculture, Abeokuta. She obtained a B.Sc degree in Mathematical Sciences (Computer Science Option) and M.Sc in Computer Science from the University of Agriculture, Abeokuta in 2000 and 2005 respectively. She is currently on her Ph.D and studies now with the Computational Intelligence Group, at the Institute of Informatics, Clausthal University of Technology, Germany; where she carries out research on Mobile Agents for E-Commerce. Her research interest include: Images and Vision, Know ledge Management, Computational Complexity, Ecommerce, Agents and Mobile Agents. She is a member of Nigeria Computer Society and has published in notable International and local Journals.

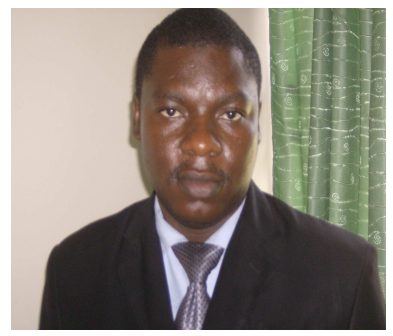

Dr. Olusegun Folorunso is a Senior Lecturer in the Department of Computer Science, University of Agriculture, Abeokuta. He obtained a B.Sc degree in Mathematical Sciences from the University of Agriculture, Abeokuta in 1992, M.Sc in Computer Science from University of Lagos in 1997 and a Ph.D in Computer Science in 2003 from the university of Agriculture, Abeokuta. His research interest includes Adoption of Information Systems strategies, Human Computer Interactions (HCI), Knowledge Management, Image Processing and Computational Intelligence . He is a member of Nigeria Computer Society and Computer Professional Registration Council of Nigeria. He has published in reputable international and local Journals. 